Document downloaded from:

http://hdl.handle.net/10251/177796

This paper must be cited as:

Ferrando-Rocher, M.; Herranz Herruzo, JI.; Sánchez-Escuderos, D.; Valero-Nogueira, A. (2020). Dual Circularly-Polarized Slot-Array Antenna in Ka-Band fed by Groove Gap Waveguide. IEEE. 421-422. https://doi.org/10.1109/IEEECONF35879.2020.9329473

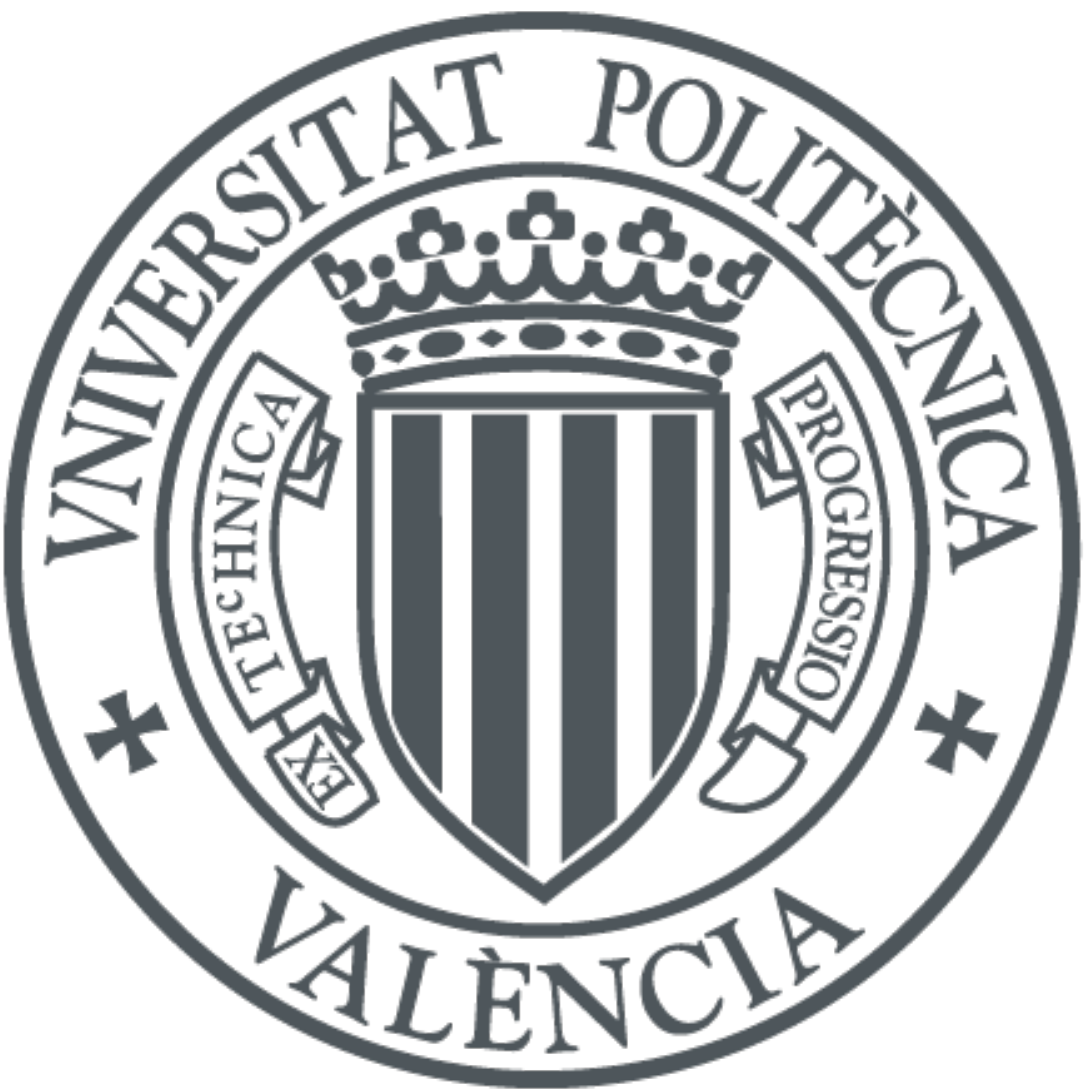

The final publication is available at

https://doi.org/10.1109/IEEECONF35879.2020.9329473

Copyright IEEE

Additional Information 


\title{
Dual Circularly-Polarized Slot-Array Antenna in Ka-Band fed by Groove Gap Waveguide
}

\author{
Miguel Ferrando-Rocher ${ }^{1,2}$, Jose I. Herranz-Herruzo ${ }^{1}$, Daniel Sánchez-Escuderos ${ }^{1}$, Alejandro Valero-Nogueira ${ }^{1}$ \\ ${ }^{1}$ Instituto de Telecomunicaciones y Aplicaciones Multimedia (iTEAM), Universitat Politècnica de València, Spain \\ ${ }^{2}$ Microwave and Applied Computational Electromagnetics Group (GMECA), Universidad de Alicante, Spain
}

\begin{abstract}
A dual circularly-polarized slot-array antenna fed by a Groove Gap Waveguide (GGW) and operating in the KaBand is presented in this paper. A simple mechanism is proposed to switch the polarization, from RHCP to LHCP, and viceversa. The lid of the antenna has two pieces: one fixed and one sliding. The fixed piece hosts T-shaped slots, and the sliding block is in charge of adjusting the offset of the perpendicular grooves with respect to the longitudinal slots. Preliminary results show an axial ratio below $1.5 \mathrm{~dB}$ for both, RHCP and LHCP, within a bandwidth of $1 \mathrm{GHz}$ centered at $30 \mathrm{GHz}$.

Index Terms-Circular polarization, Gap waveguide technology, Groove Gap Waveguide, Ka-Band, slotted-waveguide antenna
\end{abstract}

\section{INTRODUCTION}

Circular polarization is widely used in many communications systems to minimize losses associated with polarization mismatch between transmitters and receivers. The way to obtain a circularly-polarized (CP) pattern with a slotted waveguide array may be tough, but for many years remarkable designs have been achieved.

In 1957, Simmons [1] showed that a simple slot radiator formed by a pair of narrow slots crossed at proper angles, and located at the proper positions, produces a CP pattern. Since many wireless communication applications such as satellite communications use circularly polarized (CP) signals, easy switching of the polarization sense is a need.

There are few examples in the literature of two-dimensional array designs capable of switching between both circular polarization [2]-[4]. In this paper, we present a slotted waveguide array antenna with a novel switching mechanism. The antenna is formed by 12 radiating elements in an all-metallic structure. The switching between RHCP and LHCP is done by means of a sliding metallic piece.

\section{Switchable RHCP/LHCP Slot ARRAY ANTENNA DESIGN}

Basically, the array consists of 12 shunt slots, which are transformed into T-shaped slots to provide a CP pattern [5]. Fig. 1 shows a general view of the array antenna. As we can see, there is a sliding piece that can be placed in position 1 or 2. The piece is a kind of comb that adjusts the offset of the perpendicular arm of the T-shaped slot. Thereby, depending on the position of the sliding piece, an RCHP or LHCP pattern is obtained.

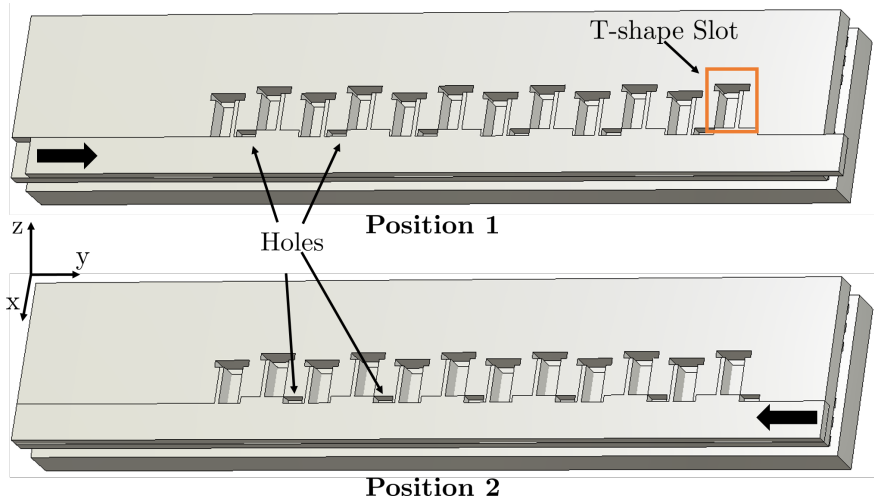

Fig. 1: 3D view of the dualCP slot array antenna.

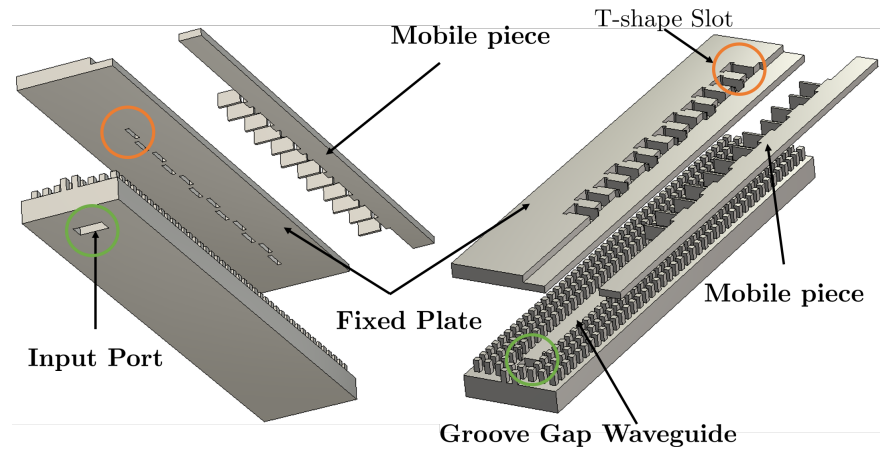

Fig. 2: Parts of the dual CP slot array antenna

Fig. 2 shows the three metal pieces that make up the antenna. Slots are fed by a Groove Gap Waveguide (GGW). It should be noted that the same concept could have been designed with a conventional rectangular waveguide (RW) but a GGW performance is quasi-identical to a RW. The advantage of using a GGW over the RW is its ability to confine the field even though the metal parts are not perfectly assembled [6][7]. Above the GGW, there is a cover that consists of two parts. One is the fixed part, where the slots are placed, and the other is the sliding piece, which controls the CP sign.

\section{Simulated Results}

The radiation pattern on the main planes of the antenna are shown in Fig. 3 at the central, upper and lower frequencies of the operation band $(29.5,30$ and $30.5 \mathrm{GHz})$. Figs. 3a and $3 \mathrm{~b}$ show the patterns if the sliding piece is in position 1 , and Figs. $3 \mathrm{c}$ and $3 \mathrm{~d}$ show the patterns if the sliding piece is in 


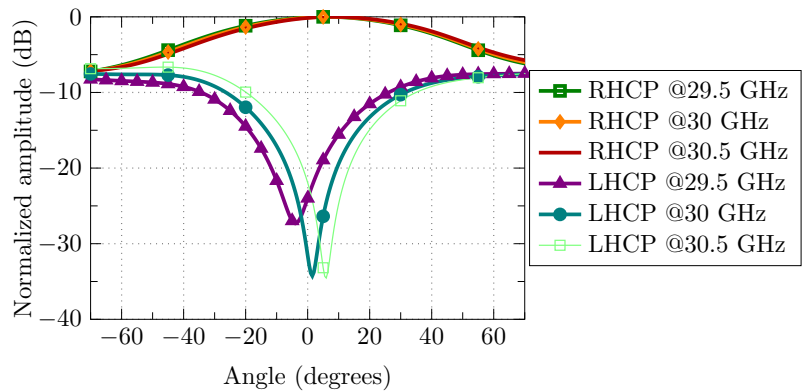

(a)

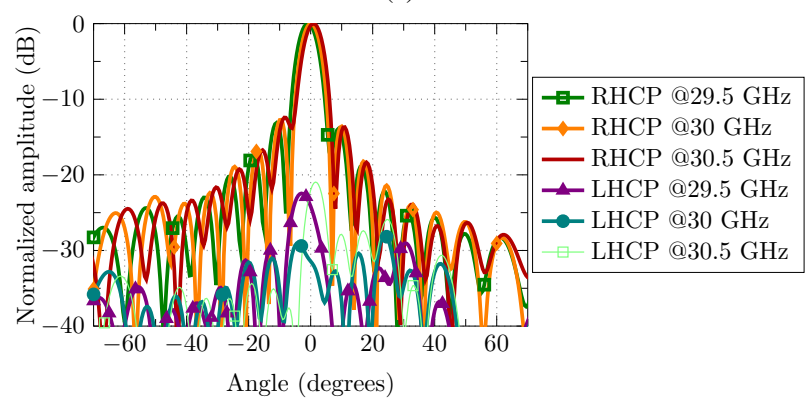

(b)

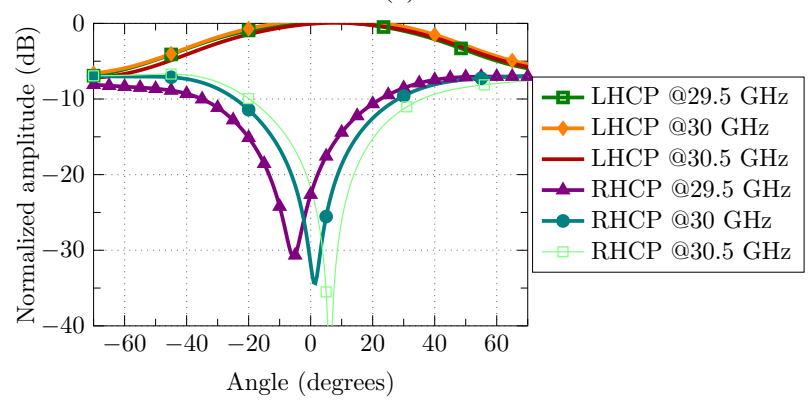

(c)

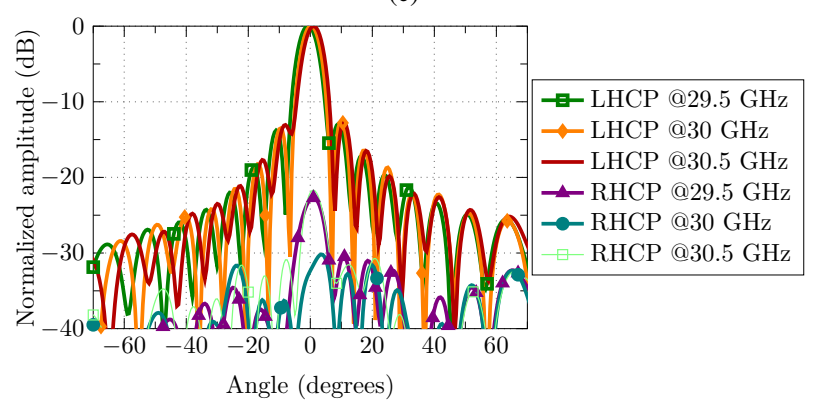

(d)

Fig. 3: Normalized simulated radiation patterns for several frequencies for both circular polarization: (a) XZ-plane (Pos. 1), (b) YZ-plane (Pos. 1), (c) XZ-plane (Pos. 2) and (d) YZ-plane (Pos. 2).

position 2. The sliding piece provides an RHCP in the first position, and an LHCP in the second position. As shown in the figures, the YZ-plane corresponds to the pattern of a 12 elements array, and the XZ-plane to the radiation pattern of a single slot.

The simulated axial ratio remains below $1.5 \mathrm{~dB}$ (see Fig. 4a) from $29.5 \mathrm{GHz}$ to $30.5 \mathrm{GHz}$. Also, it is worth noting that the

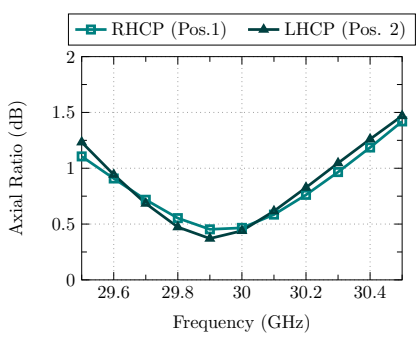

(a)

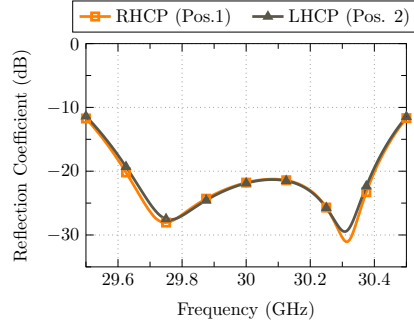

(b)
Fig. 4: (a) Simulated axial ratio. (b) Simulated reflection coefficient.

shifting of the metallic piece from position 1 to position 2 hardly affects the reflection coefficient (see Fig. 4b) of the antenna, and neither the polarization purity.

\section{CONCLUSION}

This work is based on a slot waveguide array design with circular polarization to propose a simple mechanism capable of switching between two orthogonal circular polarization. This contribution opens the appealing possibility to explore lowprofile highly-efficient and low-cost arrays with a dual circular polarization performance. An experimental validation will be carried out in the coming months.

\section{ACKNOWLEDGMENT}

This work has been supported by the Spanish Ministry of Science, Innovation and Universities (Ministerio de Ciencia, Innovación y Universidades) under project TEC2016-79700C2-1-R.

\section{REFERENCES}

[1] A. Simmons, "Circularly polarized slot radiators," IRE Transactions on Antennas and Propagation, vol. 5, no. 1, pp. 31-36, 1957.

[2] J. I. Herranz-Herruzo, A. Valero-Nogueira, M. Ferrando-Rocher, B. Bernardo, A. Vila, and R. Lenormand, "Low-cost ka-band switchable rhcp/lhcp antenna array for mobile satcom terminal," IEEE Transactions on Antennas and Propagation, vol. 66, no. 5, pp. 2661-2666, 2018.

[3] M. Ferrando-Rocher, J. I. Herranz-Herruzo, A. Valero-Nogueira, and V. M. Rodrigo, "Circularly polarized slotted waveguide array with improved axial ratio performance," IEEE Transactions on Antennas and Propagation, vol. 64, no. 9, pp. 4144-4148, 2016.

[4] A. Valero-Nogueira, J. I. Herranz-Herruzo, M. Ferrando-Rocher, R. Lenormand, A. Hirsch, and J.-L. Almeida, "Switchable rhcp/lhcp slotted waveguide array antenna for satcom on-the-move applications in ka-band," in The 8th European Conference on Antennas and Propagation (EuCAP 2014). IEEE, 2014, pp. 2047-2051.

[5] M. Ferrando-Rocher, J. I. Herranz-Herruzo, D. Sánchez-Escuderos, and A. Valero-Nogueira, "A novel circularly-polarized t-shaped slot array antenna in ka-band," in 2020 14th European Conference on Antennas and Propagation (EuCAP). IEEE, 2020, pp. 1-3.

[6] M. Ferrando-Rocher, A. Valero-Nogueira, J. I. Herranz-Herruzo, A. Berenguer, and B. Bernardo-Clemente, "Groove gap waveguides: A contactless solution for multilayer slotted-waveguide array antenna assembly," in 2016 10th European Conference on Antennas and Propagation (EuCAP). IEEE, 2016, pp. 1-4.

[7] E. Rajo-Iglesias, M. Ferrando-Rocher, and A. U. Zaman, "Gap waveguide technology for millimeter-wave antenna systems," IEEE Communications Magazine, vol. 56, no. 7, pp. 14-20, 2018. 